\title{
Combined treatment with cisplatin and sirolimus to enhance cell death in human mesothelioma
}

\author{
Mor-Li Hartman, PhD, ${ }^{\mathrm{a}}$ John Matthew Esposito, BA, ${ }^{\mathrm{a}}$ Beow Yong Yeap, $\mathrm{ScD},{ }^{\mathrm{b}}$ and \\ David John Sugarbaker, MD ${ }^{\mathrm{a}}$
}

Objective: Although platinum-based chemotherapy is widely used in malignant pleural mesothelioma, its modest therapeutic effect warrants identification of enhancing agents. As with many cancers, the phosphatidylinositol 3-kinase/Akt pathway is often activated in malignant pleural mesothelioma and has been implicated in the tumor's aggressiveness. Sirolimus is a well-established inhibitor of the mammalian target of rapamycin. We sought to determine whether combination treatment with sirolimus and cisplatin would enhance cell death in malignant pleural mesothelioma.

Methods: Human malignant pleural mesothelioma cell lines were incubated with sirolimus or cisplatin alone or in combination and assayed for cell viability. To characterize phosphorylation status after treatment, Akt and downstream proteins of mammalian target of rapamycin pathway, p70 S6 kinase and 4E-BP1, were analyzed by Western blot. Effect of combination treatment was also analyzed with extreme drug resistance assay in 12 human malignant pleural mesothelioma tumors with varying resistance to cisplatin.

Results: Individual malignant pleural mesothelioma cell lines exhibited a range of sensitivities to each drug without correlation with subtype. Sirolimus and cisplatin significantly $(P=.029)$ increased cell death versus either drug alone in 4 cell lines. Combined treatment caused dephosphorylation of Akt, 4E-BP1, and p70 S6 kinase. Cell proliferation was significantly decreased in tumors subjected to sirolimus and cisplatin versus cisplatin or sirolimus alone.

Conclusions: Sirolimus appears to enhance the cytotoxicity of cisplatin in malignant pleural mesothelioma cell lines through the mammalian target of rapamycin pathway. These results provide a basis for the clinical evaluation of combined sirolimus and cisplatin chemotherapy in malignant pleural mesothelioma. (J Thorac Cardiovasc Surg 2010;139:1233-40)

Malignant pleural mesothelioma (MPM) is a mesodermally derived neoplastic disease that arises in the pleura and kills through direct invasion of surrounding structures. It affects approximately 3000 patients in the United States annually. ${ }^{1}$ There are 3 distinct histologic subtypes: epithelial, sarcomatoid, and biphasic (a mixed type that contains both an epithelial and a sarcomatoid component). ${ }^{1,2}$ The pathogenesis of MPM is not fully understood. A highly aggressive cancer, MPM responds poorly to treatment with platinum-based combination chemotherapy, with a median survival of up to 12 months. Multimodality treatment with cytoreductive surgery, followed by a combination of chemotherapy and radiation therapy, has been shown to prolong survival for

\footnotetext{
From the Novel Therapeutics Laboratory, Division of Thoracic Surgery, Brigham and Women's Hospital, ${ }^{\text {a }}$ and the Hematology-Oncology Unit, Department of Medicine, Massachusetts General Hospital, ${ }^{\mathrm{b}}$ Harvard Medical School, Boston, Mass.

Disclosures: None.

Supported by the International Mesothelioma Program, Brigham and Women's Hospital, Boston, Mass.

Received for publication April 16, 2009; accepted for publication June 21, 2009; available ahead of print Oct 23, 2009.

Address for reprints: David J. Sugarbaker, MD, The Richard E. Wilson Professor of Surgical Oncology, Chief, Division of Thoracic Surgery, Brigham and Women's Hospital, 75 Francis St, Boston, MA 02115 (E-mail: dsugarbaker@partners.org). 0022-5223/\$36.00

Copyright (c) 2010 Published by Elsevier Inc. on behalf of The American Association for Thoracic Surgery

doi:10.1016/j.jtcvs.2009.06.027
}

select patients with early-stage disease. ${ }^{1}$ Although platinumbased chemotherapy is widely used, it has a modest therapeutic effect, and the identification of new agents to enhance its therapeutic effect is warranted.

Cisplatin is a DNA-damaging agent used to treat a wide range of tumor types. Apoptosis is triggered when the damage done by cisplatin becomes irreversible. The major limitation of cisplatin is its narrow therapeutic window. At high doses it becomes nephrotoxic and ototoxic, whereas at low doses the affected DNA is capable of self-repair. ${ }^{3,4}$ Cisplatin has been studied as a single agent in patients with diffuse MPM and demonstrated an overall response rate of $14 \%$ when given daily at a dose of $100 \mathrm{mg} / \mathrm{m}^{2}{ }^{5}$ Cisplatin alone was included as the control arm of 2 phase III trials of 2 novel agents: pemetrexed in combination with cisplatin ${ }^{6}$ and cisplatin in combination with raltitrexed. ${ }^{5,7}$ Combined chemotherapeutic regimens consisting of cisplatin and doxorubicin and of cisplatin and mitomycin have been tested, and moderate antitumor activity was observed with both regimens. ${ }^{8}$ Finally, Vogelzang and colleagues ${ }^{6}$ conducted a phase III trial to determine whether combination treatment with pemetrexed and cisplatin would improve survival relative to cisplatin alone. In that study, median survival was 12.1 months in the pemetrexed and cisplatin arm versus 9.3 months in the control (cisplatin alone) arm. Better 


\author{
Abbreviations and Acronyms \\ 4E-BP1 = eukaryotic translation initiation factor \\ $4 \mathrm{E}$ binding protein 1 \\ Akt $=$ protein kinase $\mathrm{B}$ \\ DMSO $=$ dimethyl sulfoxide \\ MPM = malignant pleural mesothelioma \\ mTOR $=$ mammalian target of rapamycin \\ RPMI = Roswell Park Memorial Institute \\ medium \\ Thr $=$ threonine
}

have been observed. ${ }^{15}$ In this study, we demonstrate that combined exposure to sirolimus and cisplatin significantly increases cell death in MPM cell lines relative to either drug alone. We characterize the phosphorylation statuses of Akt, p70 S6 kinase, and 4E-BP1 after each treatment. Finally, we show that cell growth is significantly inhibited in human MPM tumors by sirolimus and cisplatin treatment as relative to either cisplatin or sirolimus alone.

\section{MATERIALS AND METHODS Cell lines}

MPM cell lines MS257 (epithelial), MS924 (epithelial), MS428 (sarcomatoid), and MS589 (biphasic) were provided by Jonathan A. Fletcher from the Department of Pathology, Brigham and Women's Hospital. MPM cell line H2052 (epithelial) was provided by Raphael Bueno and Gavin J. Gordon from the Division of Thoracic Surgery, Brigham and Women's Hospital. ${ }^{18}$ MPM cell line JMN1B (epithelial) was provided by Dr. James Rheinwald, Department of Dermatology, Brigham and Women's Hospital. ${ }^{19}$ All 6 cell lines were maintained in Roswell Park Memorial Institute medium (RPMI) 1640 (Gibco, Carlsbad, Calif) supplemented with $15 \%$ fetal bovine serum (HyClone, Logan, Utah), 100-U/mL penicillin, 100- $\mu \mathrm{g} /$ $\mathrm{mL}$ streptomycin, $0.25-\mu \mathrm{g} / \mathrm{mL}$ amphotericin B (Gibco), and 2-mmol/L glutamine (Gibco).

Photographs of the cells were taken with the Nikon Eclipse TE2000-S microscope, the SPOT Insight QE camera (Diagnostic Instruments, Sterling Heights, Mich), and the SPOT Advanced computer program. The $20 \times \mathrm{ob}-$ jective lens was used with a $10 \times$ eyepiece. In all experiments, cells were seeded 24 hours before treatment. All of the experiments were repeated at least 3 times.

\section{Drugs}

Sirolimus in dimethyl sulfoxide (DMSO) was purchased from EMD (EMD Biosciences, Inc, San Diego, Calif). The drug was diluted in fresh medium without serum before each experiment. Cisplatin (cis-diammineplatinum[II] dichloride) was purchased from Sigma (Saint Louis, Mo). The drug was diluted in medium without serum for the stock solution.

\section{Proliferation Assay for Cisplatin Dose-Response Curve}

MPM cell lines were plated in 96-well tissue culture plates; 1700 to 2500 cells (depending on cell line) per well were cultured in RPMI 1640 containing $15 \%$ fetal bovine serum overnight. Cells were incubated for 24 hours with cisplatin (range, $1-500 \mu \mathrm{mol} / \mathrm{L}$ ) or without cisplatin (control). After 24 hours, cells were washed twice with RPMI 1640, and cell viability was analyzed with the CellTiter 96 aqueous nonradioactive cell proliferation assay (Promega, Madison, Wis). Experiments were performed at $37^{\circ} \mathrm{C}$, with 4 wells for each concentration and each cell line. The average and SD were calculated from 4 wells. Experiments were performed in triplicate on at least 2 separate occasions. Results are expressed as number of viable cells relative to control (untreated cells) for each cell line within an experiment. The relative number of viable control cells thus equals 1 for each cell line. The active washout of drugs in all these experiments was selected to mimic the washout of drug after a 1-hour chemotherapeutic intracavitary lavage in patients with MPM undergoing surgery.

\section{Proliferation Assay for Sirolimus Dose-Response Curve}

Cells were seeded as described for cisplatin experiments. The sirolimus experiments differed in three respects. (1) The concentration range for sirolimus was 1 to $500 \mathrm{nmol} / \mathrm{L}$ (rather than $1-500 \mu \mathrm{mol} / \mathrm{L}$ ). (2) A control with DMSO concentration matched to the samples was used. (3) The drug was 
washed out after 24 hours of incubation, and the proliferation assay was performed after washout and every 24 hours subsequently for a total of 96 hours. Drug washout was designated time zero.

\section{Proliferation Assay for Cisplatin-Sirolimus Combination Treatment Dose-Response Curve}

Cells were seeded as described for cisplatin and sirolimus individual experiments. Cells were incubated for 24 hours with $100-\mathrm{nmol} / \mathrm{L}$ sirolimus, $50-\mu \mathrm{mol} / \mathrm{L}$ cisplatin, and both drugs. A control with DMSO concentration matched to the samples was used. After 24 hours, the cells were washed twice with phosphate-buffered saline solution, and cell proliferation was recorded at 0,24 and 48 hours. All other conditions were identical to the previous experiments. A $50-\mu \mathrm{mol} / \mathrm{L}$ concentration of cisplatin was used because it was the lowest cisplatin concentration that still yielded significant cell death (Figure 1), and a 100-nmol/L concentration of sirolimus was used because that dose yielded the maximal response (Figure 2).

\section{Antibodies and Western Blotting}

H2052 and MS428 cell lines were cultured overnight in RPMI 1640 containing $15 \%$ fetal bovine serum. Cells $\left(1.3-1.7 \times 10^{6}\right.$ cells/flask $)$ were incubated for 24 hours with indicated concentrations of either 100-nmol/L sirolimus, $50-\mu \mathrm{mol} / \mathrm{L}$ cisplatin, or both drugs. Cells that were treated with matched DMSO concentration served as the control preparation. After 24 hours, cells were washed twice with phosphate-buffered saline solution and incubated with medium for an additional 24 hours. Cells were then lysed in lysis buffer $(50-\mathrm{mmol} / \mathrm{L}$ tris[hydroxymethyl]aminomethane chloride $\mathrm{pH}$ $8.0,100-\mathrm{mmol} / \mathrm{L}$ sodium fluoride, $30-\mathrm{mmol} / \mathrm{L}$ sodium pyrophosphate, 2-mmol/L sodium molybdate, $5-\mathrm{mmol} / \mathrm{L}$ ethylenediaminetetraacetic acid, 2 -mmol/L sodium vanadate, and $1 \%$ Tergitol-type NP-40) containing protease inhibitor cocktail (Sigma). Proteins were separated by gel electrophoresis on $4 \%$ to $12 \%$ tris(hydroxymethyl)aminomethane/glycine polyacrylamide gels (Invitrogen, Carlsbad, Calif), transferred to nitrocellulose membranes (Bio-Rad, Hercules, Calif), and detected by immunoblotting with an enhanced chemiluminescence system (Boston Bioproducts, Worcester, Mass). Antibodies for phosphorylated 4E-BP1 threonine (Thr) 70, phosphorylated 4E-BP1 Thr 37/46, phosphorylated 70 S6 kinase Thr 389, and phosphorylated Akt Thr 308 were purchased from Cell Signaling Technology (Beverly, Mass). Glyceraldehyde 3-phosphate dehydrogenase antibody was purchased from Ambion (Austin, Tex). ECL anti-rabbit IgG horseradish peroxidaselinked species-specific whole antibody and ECL anti-mouse IgG horseradish peroxidase-linked species-specific whole antibody were purchased from Amersham Bioscience (GE Healthcare, Piscataway, NJ). The protein levels were studied 24 hours (as a middle time window between $0-48$ hours) after the drugs were washed out because for MS428 the strongest effect of the cisplatin and sirolimus treatment was observed after 48 hours.

\section{Extreme Drug Resistance Assay}

Under an institutional review board-approved protocol, 12 tumor specimens removed during cytoreductive surgery for MPM were analyzed for extreme drug resistance and intermediate resistance in a cellular proliferation assay described previously. ${ }^{20,21}$

\section{Statistical Analysis}

Each dose-response curve was characterized by a 4-parameter logistic model estimated by nonlinear regression analysis with GraphPad Prism 4 software (GraphPad Software, Inc, San Diego, Calif). In the absence of treatment with cisplatin or sirolimus, the control dose was set arbitrarily 2 logs below the lowest drug concentration, and control cell viability was set to reflect $100 \%$ normal proliferation. Nonlinear regression analysis with log-transformed data was used to determine half maximal inhibitory concentration $\left(\mathrm{IC}_{50}\right)$ value (theoretical concentration required to inhibit $50 \%$ of cells) as a relative measure of drug sensitivity. The 2 -sample $t$ test based on an assumption of unequal variances was used with the

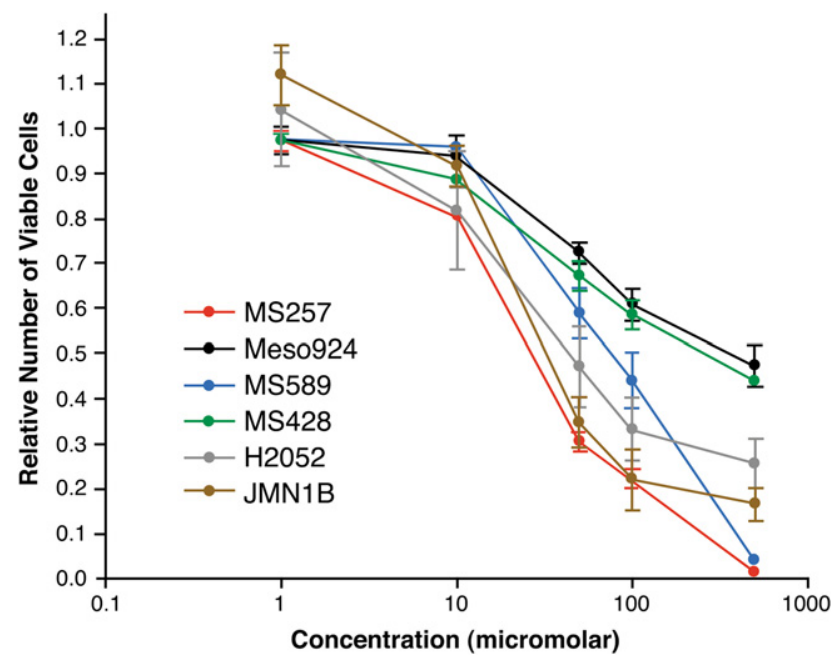

FIGURE 1. Cisplatin dose-response curves in malignant pleural mesothelioma cell lines. Effect of loss of cell viability detected with different doses of cisplatin (1-500- $\mu \mathrm{mol} / \mathrm{L})$ in 6 malignant pleural mesothelioma cell lines. Results represent mean of at least 3 experiments; bars represent SD.

Satterthwaite approximation to compare the difference in loss of cell viability between 2 treatment groups in each cell line. Representative experiments typically correspond to the median of the outcome range among the repeated experiments. Experiments were repeated 3 times. The differences in cell growth inhibition in tumor specimens treated with cisplatin alone versus the combination treatment were analyzed with a paired $t$ test. The same analysis was performed for tumors treated with sirolimus alone versus the combination treatment. All $P$ values were based on a 2-sided hypothesis and were computed by SAS 9.1 software (SAS Institute, Inc, Cary, NC).

\section{RESULTS}

\section{Effect of Cisplatin on Cell Viability}

The relationship between concentration of cisplatin (range, $1-500 \mu \mathrm{mol} / \mathrm{L}$ ) and loss of cell viability in MPM cell lines (MS924, MS428, H2052, MS589, MS257, and JMN1B) is depicted in Figure 1. The cell lines expressed varying sensitivities to cisplatin, falling into 2 groups with one relatively more sensitive than the other. Nonlinear regression analysis of survival curves produced an excellent fit for all cell lines $\left(R^{2}=0.8725-0.9870\right)$ except for H2052. Extrapolated $\mathrm{IC}_{50}$ values showed that 4 of the cell lines (MS257, JMN1B, H2052, and MS589) were relatively more sensitive to cisplatin ( $\mathrm{IC}_{50}$ range, $21.06-97.79 \mu \mathrm{mol} /$ L) than were the other 2 (MS924 and MS428), which were relatively more resistant to cisplatin. We could not reliably calculate the $\mathrm{IC}_{50}$ values from this analysis, because the curves ended very close to the $50 \%$ mark of relative cell viability. The graph in Figure 1, however, shows that the $\mathrm{IC}_{50}$ of MS924 and MS428 was higher than the $\mathrm{IC}_{50}$ of MS589.

\section{Effect of Sirolimus on Cell Viability}

The relationship between sirolimus concentration (range, $1-500 \mathrm{nmol} / \mathrm{L}$ ) and loss of cell viability is shown in Figure 2. The data revealed that different cell lines have different 

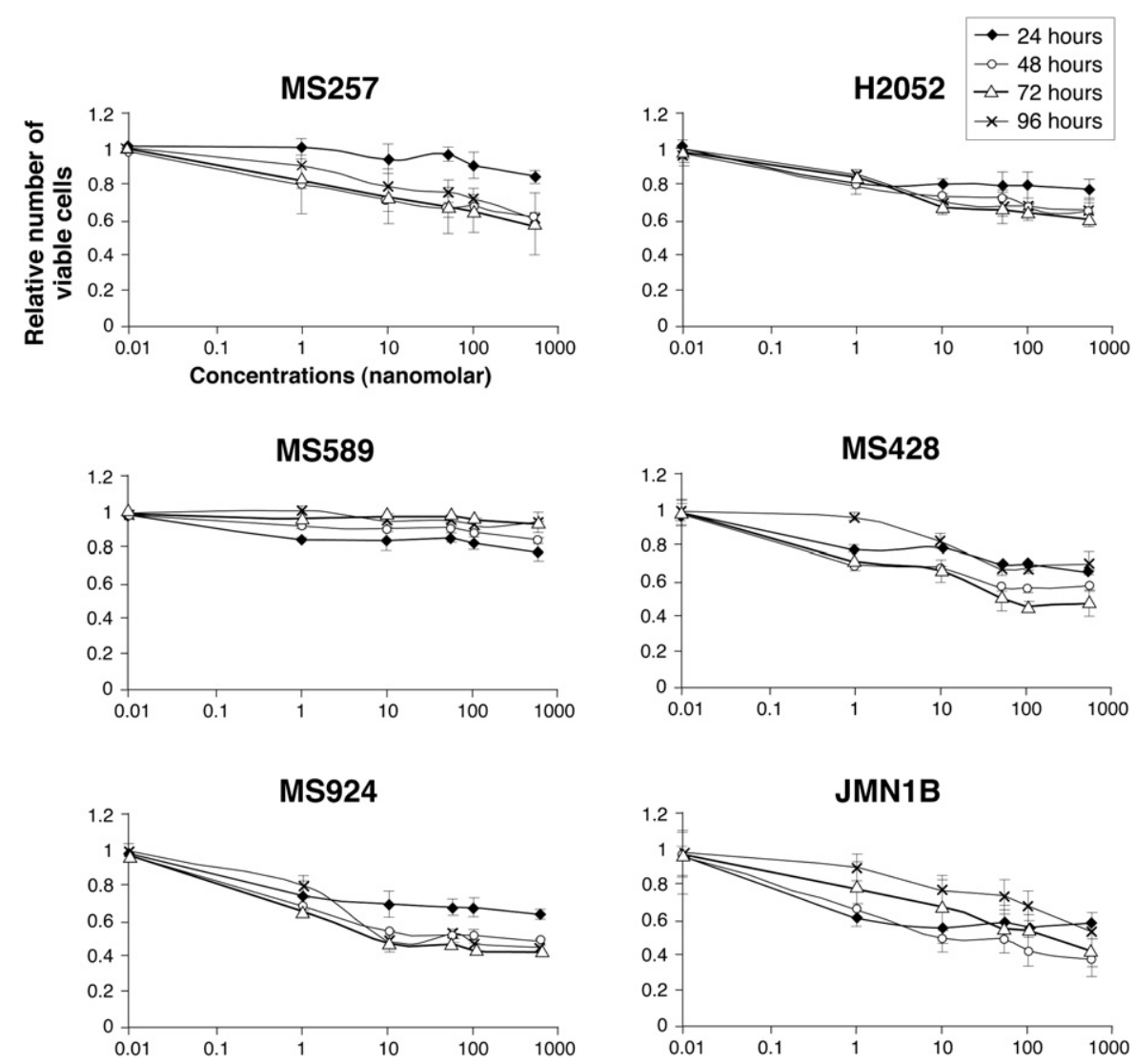

FIGURE 2. Sirolimus dose-response curves in malignant pleural mesothelioma cell lines. Effect of loss of cell viability detected with different doses of sirolimus (1-500 nmol/L) and time courses in 6 malignant pleural mesothelioma cell lines. Results represent mean of at least 3 experiments; bars represent SD.

sensitivities to sirolimus. Loss of cell viability with sirolimus treatment was time dependent. Four of 6 cell lines responded maximally 72 hours after the drug was washed out. At least 3 of 6 cell lines responded maximally to the $100-\mathrm{nmol} / \mathrm{L}$ dose of sirolimus.

\section{Effect of Combined Treatment With Sirolimus and Cisplatin on Cell Viability}

The effect of combination treatment with $100-\mathrm{nmol} / \mathrm{L}$ sirolimus and $50-\mu \mathrm{mol} / \mathrm{L}$ cisplatin is depicted in Figure 3. This effect was stronger than that of either sirolimus or cisplatin treatment alone. Figure 3 summarizes the data derived from the 3 test conditions (plus control) across all cell lines: sirolimus alone, cisplatin alone, and combined sirolimus and cisplatin. The MS589 and MS428 cell lines demonstrated $91.2 \%(P<.0001)$ and $45.1 \%(P=.0002)$ losses of cell viability, respectively, with combined sirolimus and cisplatin and with cisplatin alone 48 hours after the drugs were washed out. JMN1B and MS924 demonstrated $46.6 \%(P=.0010)$ and $34.9 \%(P=.0009)$ reductions in cell viability, respectively, with combined treatment and with cisplatin alone at time zero. Loss of cell viability with combined treatment in the MS257 and H2052 cell lines did not reach statistical significance relative to values for cisplatin alone. Those cell lines demonstrated relatively high sensitivity to cisplatin alone (Figure 1).

\section{Effects of Sirolimus, Cisplatin, and Combined Sirolimus and Cisplatin on Akt and Proteins Downstream to mTOR}

We sought to determine whether Akt and downstream proteins of mTOR were modulated by treatment with sirolimus, cisplatin, and the combined regimen. First, with Western blot analysis, we analyzed phosphorylation status after each treatment in 2 cell lines (MS428 and H2052) that demonstrated reciprocal sensitivity to each drug. Akt was dephosphorylated at Thr 308 by cisplatin alone and the combined sirolimus and cisplatin treatment in both cell lines (Figure 4); however, sirolimus alone increased the phosphorylation of Akt Thr 308 relative to the control cells. Both cell lines (Figure 4) exhibited high p70 S6 kinase dephosphorylation at Thr 389 both with sirolimus alone and with sirolimus and cisplatin together. Phosphorylation of p70 S6 kinase at Thr 389 was observed both in the control preparation and with cisplatin treatment alone, indicating that cisplatin does not affect the phosphorylation of this kinase. 

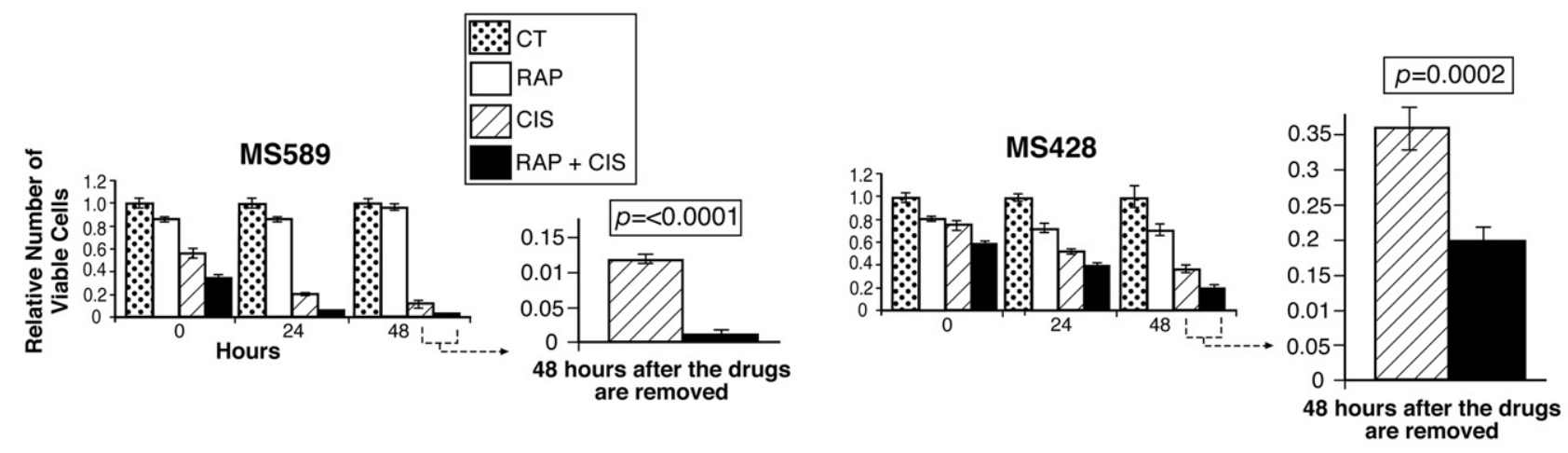

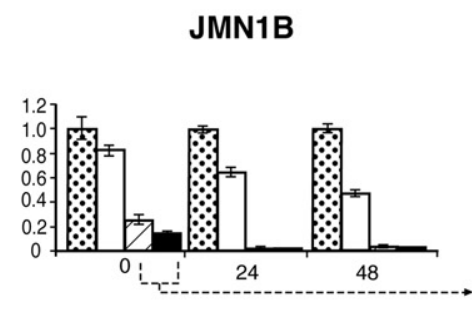

A
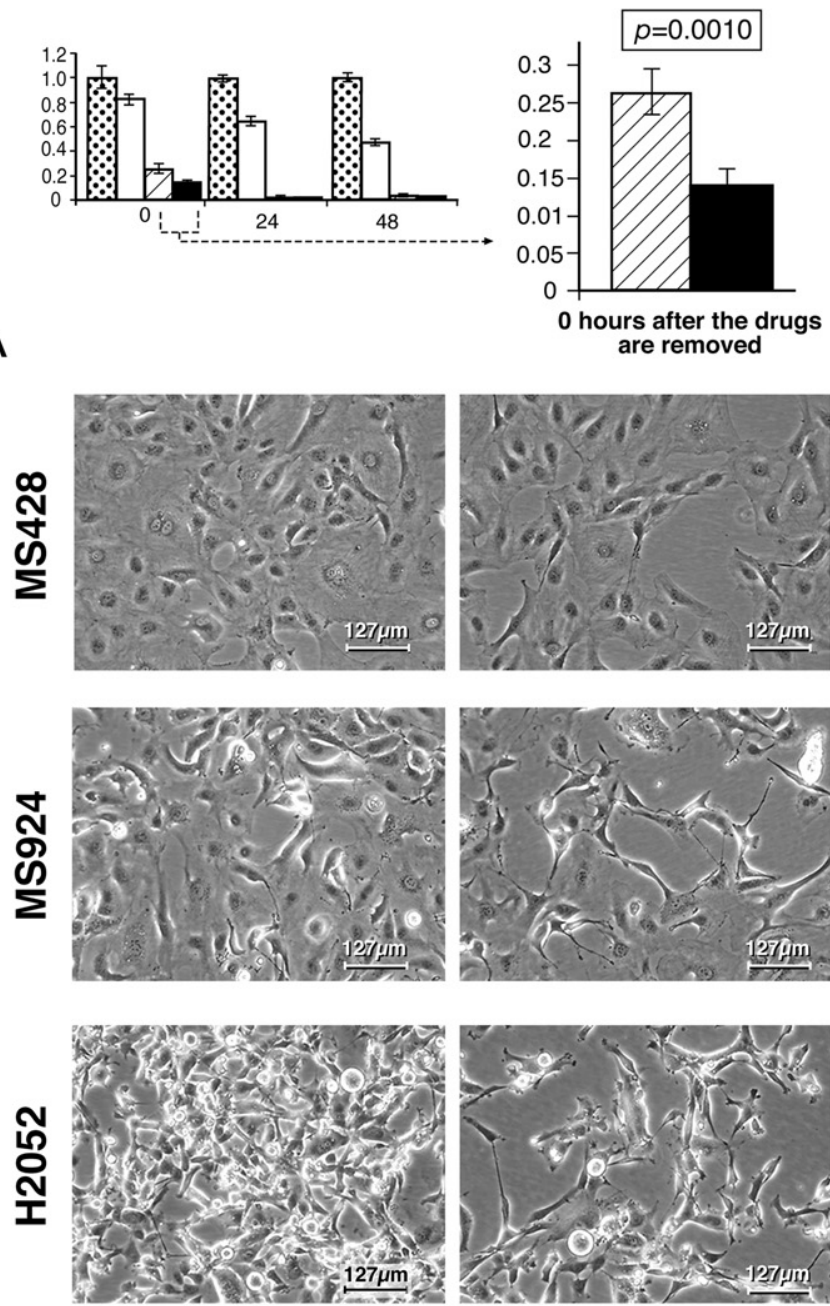

B
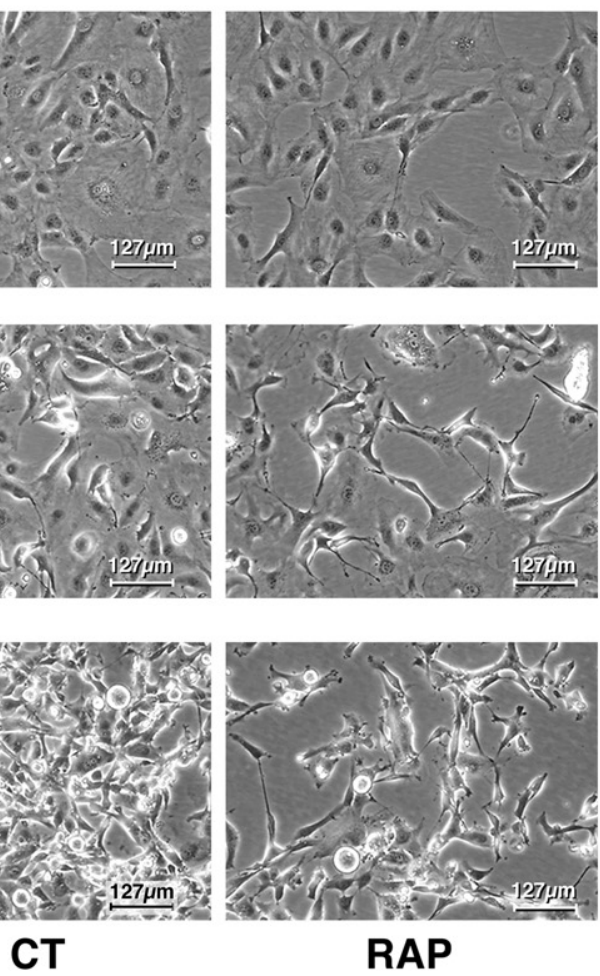
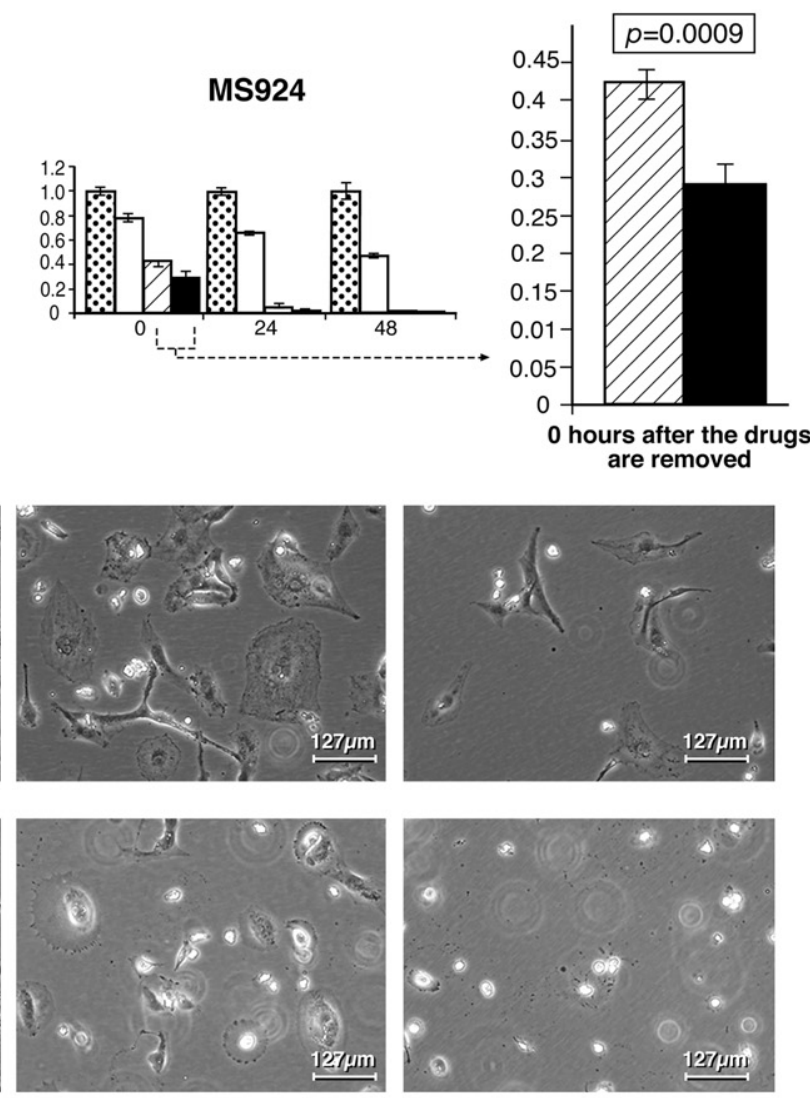

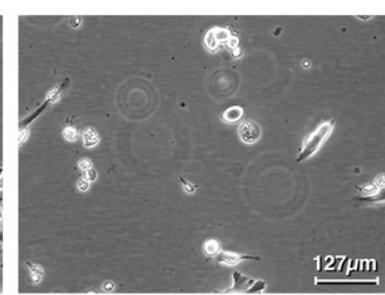

CIS

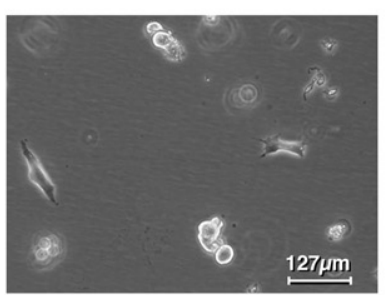

RAP + CIS

FIGURE 3. Sirolimus and cisplatin combination in malignant pleural mesothelioma cell lines. A, Effects of loss of cell viability in different malignant pleural mesothelioma cell lines (MS589, MS428, JMN1B, and MS924) with sirolimus (RAP), cisplatin (CIS), combination of both drugs $(R A P+C I S)$, and nontreatment control $(C T)$ of dimethyl sulfoxide vehicle. For each cell line, enlarged figure near each graph shows increased loss of cell viability from both drugs versus cisplatin alone at specific time points. Columns represent mean of 4 wells; bars represent SD. Each $P$ value is presented in box. B, Malignant pleural mesothelioma cell lines MS428, MS924, and H2052 were treated as in part A and photographed 24 hours after drugs were washed out. Original magnification $200 \times$; abbreviations as in part A. 


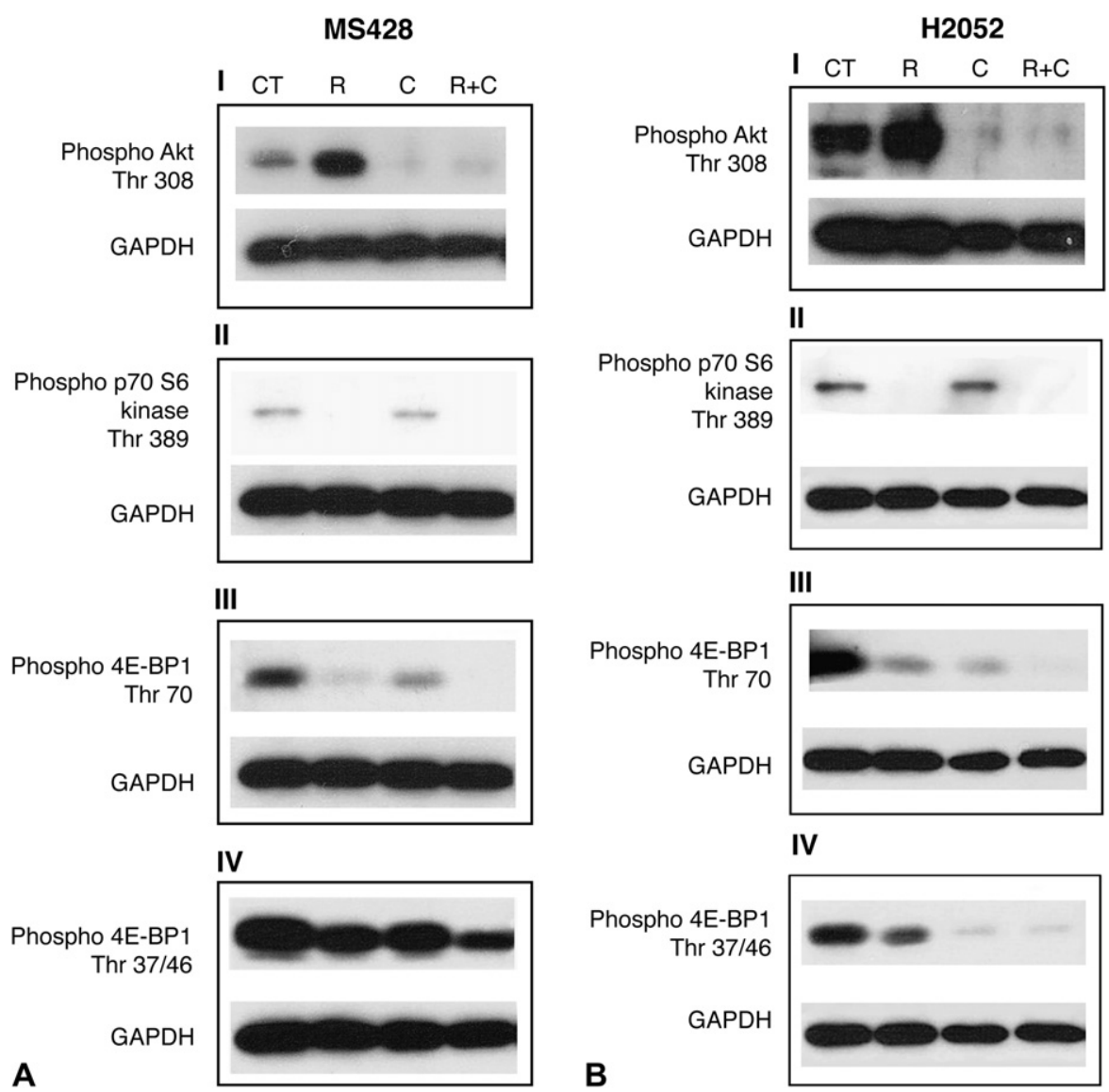

FIGURE 4. Dephosphorylation of Akt, p70 S6 kinase, and 4E-BP1 after sirolimus and cisplatin combined treatment. Western blot analysis demonstrates phosphorylation status of the Akt/mTOR pathway proteins using specific antibodies (Phospho) against specific phosphorylation sites (I-IV) of the indicated proteins. Column $A$ represents malignant pleural mesothelioma cell line MS428. Column $B$ represents malignant pleural mesothelioma cell line H2052. Both cell lines were treated with sirolimus $(R)$, cisplatin $(C)$, and the combination of both drugs $(R+C)$. Untreated cells in vehicle (dimethyl sulfoxide) were used as control $(C T)$. Glyceraldehyde 3-phosphate dehydrogenase $(G A P D H)$ was used as loading control.

Both cell lines (Figure 4) exhibited moderate dephosphorylation of 4E-BP1 at Thr 70 with both sirolimus alone and cisplatin alone but showed virtually complete dephosphorylation of 4E-BP1 with combined sirolimus and cisplatin. We observed a different pattern of phosphorylation with a different phosphorylated 4E-BP1 antibody targeted to phosphorylation site Thr 37/46. MS428 (Figure 4) exhibited slight dephosphorylation after treatment both with sirolimus alone and with cisplatin alone, whereas slightly greater dephosphorylation was observed with sirolimus and cisplatin combined. H2052 produced similar results in terms of sirolimus treatment with the same antibody to Thr 37/46 (Figure 4); however, we observed significant dephosphorylation of 4E-BP1 with cisplatin alone and with both drugs relative to MS428 under the same conditions.

\section{Effect of Combined Treatment With Sirolimus and Cisplatin on Mesothelioma Tumor Specimens}

To obtain a clearer indication of the efficacy of the sirolimus and cisplatin combination, we tested 12 tumor speci- mens with the cellular proliferation assay (extreme drug resistance assay). Tumor specimens exhibiting varying resistances to cisplatin were treated with combined sirolimus and cisplatin and compared with cisplatin alone. Percentage cell growth inhibition was significantly higher when the 12 tumor specimens were exposed to the sirolimus and cisplatin combination compared to cisplatin alone $(P=.0002$; Table 1$)$ A similar increase in cell growth inhibition was observed with the sirolimus and cisplatin combination compared with sirolimus alone $(P=.0133$; Table 1$)$.

\section{DISCUSSION}

In these experiments, combined treatment with sirolimus and cisplatin elicited greater cell death in MPM cell lines than did either drug alone. Combined treatment caused greater dephosphorylation of 4E-BP1 than did either drug alone and caused dephosphorylation of Akt and p70 S6 kinase. Finally, in 12 MPM tumor specimens, we demonstrated that cell growth inhibition is greater after combined sirolimus and cisplatin treatment than after either drug alone. 
TABLE 1. Inhibition by cellular proliferation assay after each treatment

\begin{tabular}{lcc}
\hline & Cisplatin & Sirolimus \\
\hline $\begin{array}{l}\text { No. of specimens } \\
\text { Average percent inhibition } \\
\text { after single-drug treatment } \\
\quad \text { average and range) }\end{array}$ & $61 \%(34 \%-87 \%)$ & 12 \\
$\begin{array}{l}\text { Average percent inhibition } \\
\text { after combined therapy } \\
\quad \text { average and range })\end{array}$ & $84 \%(68 \%-98 \%)$ & $84 \%(68 \%-98 \%)$ \\
$\begin{array}{l}\text { Average increase in } \\
\text { percent inhibition with } \\
\text { combination (average } \\
\text { and range) }\end{array}$ & $23 \%(2 \%-44 \%)$ & $20 \%(-6 \%-74 \%)$ \\
$P$ value* & & \\
\hline
\end{tabular}

*The $t$ test was performed to compare inhibition between single-drug treatment and combined therapy.

The proliferation assays conducted on each cell line revealed varying sensitivities, a finding that has been shown previously by Gordon and colleagues, ${ }^{18}$ and there was no correlation between drug sensitivity and cell type. Interestingly, at least in 3 of 6 cell lines, cisplatin sensitivity was inversely related to sirolimus sensitivity. For at least 3 of the cell lines, the maximum effect was achieved at the 100$\mathrm{nmol} / \mathrm{L}$ dose of sirolimus. Sawyers ${ }^{22}$ has suggested that if more than $1-\mathrm{nmol} / \mathrm{L}$ of sirolimus is required to achieve the desired cell effect, then it is likely that other pathways in addition to mTOR are probably involved, which justifies the use of the combined regimen in our study.

In our study, the combination sirolimus and cisplatin significantly increased cell death in 4 MPM cell lines compared with either drug alone. For 2 cell lines (MS428 and MS589), this effect was time dependent.

Sirolimus inhibits mTOR, induces insulin receptor substrate 1 expression, and abrogates feedback inhibition of the pathway, resulting in Akt activation. ${ }^{23} \mathrm{We}$ also observed Akt activation in 2 MPM cell lines treated with sirolimus. Cisplatin alone or combined with sirolimus resulted in dephosphorylation of Akt.

Sirolimus leads to dephosphorylation and inactivation of p70 S6 kinase. ${ }^{15}$ In our study, cell lines MS428 and H2052 when treated with sirolimus alone or combined sirolimus and cisplatin, displayed dephosphorylation of p70 S6 kinase.

Our work with the MS428 and H2052 cell lines subjected to sirolimus treatment shows that the 4E-BP1 Thr 37/46 site is less sensitive to sirolimus, whereas Thr 70 is highly sensitive to sirolimus, as reported by others. ${ }^{24}$ Dephosphorylation of 4E-BP1 (Thr 70 in both cell lines and Thr 37/46 in MS 428 cell line) after combination treatment with cisplatin and sirolimus was greater than with either drug alone. Cisplatin treatment also moderately dephosphorylated 4E-BP1 at Thr 70 in both cell lines, but the dephosphorylation effect at $\mathrm{Thr} 37 / 46$ was strong only in the H2052 cell line. Whether sensitivity to cisplatin correlates with the ability of Thr 37/46 to be dephosphorylated merits further study.

Preliminary results of the cellular proliferation assay in 12 MPM tumor specimens support our in vitro findings. In the tumor specimens, exposure to combined sirolimus and cisplatin significantly increased the inhibition of cell growth relative to cisplatin alone or sirolimus alone. Of interest, the increase in cell growth inhibition with the combination treatment is significant over a range of sensitivity to cisplatin alone. These data provide additional support for the sirolimus and cisplatin combination in the treatment of MPM.

Other in vitro studies of sirolimus and cisplatin in different types of cancer have shown that sirolimus enhances cell death and increases sensitivity to cisplatin., ${ }^{3,14}$ The mechanism by which sirolimus increases the sensitivity of cisplatin is not clear; however, several reports have highlighted the involvement of different pathways. It is important to note that these analyses have been performed on different cell lines, at different concentrations of cisplatin and sirolimus, and with different sirolimus derivates, and all these factors may influence the results. Yan and coworkers ${ }^{17}$ have reported that activation of Akt and dephosphorylation of 4E-BP1, with attendant decrease in free eukaryotic translation initiation factor $4 \mathrm{E}$ levels and cap-dependent translation, mediates the proapoptotic effect of sirolimus. Therefore, eukaryotic translation initiation factor $4 \mathrm{E}$ is a key factor in promoting tumorigenic activity and antiapoptotic activity. ${ }^{16}$ Our data revealed similar effects of complete dephosphorylation of 4E-BP1 Thr 70 with the combined regimen relative to either drug alone. From our results, it appears that inhibition of p70 S6 kinase may be involved in the sirolimus chemosensitivity mechanism. In addition, the function of S6 kinase, which is downstream to p70 S6 kinase, has been related to apoptotic resistance of mesothelioma cells in 3-dimensional culture and tumor fragment spheroids. ${ }^{25}$ Further investigation will be required to elucidate other pathways that are involved in the molecular mechanism of this combination of drugs in MPM cell lines.

To our knowledge, this is the first demonstration of the effect of combined sirolimus and cisplatin chemotherapy on human MPM cell lines and tumor specimens. The enhanced cytotoxic effect of the combined regimen relative to either drug alone has important clinical implications when one considers the current limitations of cisplatin chemotherapy. The dephosphorylation effect of combined treatment on Akt, 4E-BP1, and P70 S6 kinase supports the hypothesis that sirolimus increases cisplatin cytotoxicity through the mTOR pathway. Our views are further supported by the inhibitory effect of this treatment on cell growth in human MPM tumors, as evidenced by cellular proliferation assay (extreme drug resistance). In our view, the effects of the sirolimus and cisplatin combination demonstrated in this study provide ample justification for pursuing this treatment in the clinical setting of trimodality therapy for MPM. 
We acknowledge Raphael Bueno, Jonathan Fletcher, Gavin Gordon, James Rheinwald, and William Richards for their assistance in this research effort. Yen Huynh and Lilian Tee provided technical assistance with the extreme drug resistance assay. We also acknowledge Ann S. Adams for editorial work and Marcia Williams for assistance with the graphic presentation.

\section{References}

1. Sugarbaker DJ, Garcia JP, Richards WG, Harpole DH Jr, Healy-Baldini E, DeCamp MM Jr, et al. Extrapleural pneumonectomy in the multimodality therapy of malignant pleural mesothelioma. Results in 120 consecutive patients. Ann Surg. 1996;224:288-96.

2. Corson JM, Renshaw AA. Pathology of mesothelioma. In: Aisner J, Arriagada R, Green M, Martini N, Perry M, eds. Comprehensive textbook of thoracic oncology. Baltimore (MD): Williams and Wilkins; 1996:757-8.

3. Beuvink I, Boulay A, Fumagalli S, Zilbermann F, Ruetz S, O'Reilly T, et al. The mTOR inhibitor RAD001 sensitizes tumor cells to DNA-damaged induced apoptosis through inhibition of p21 translation. Cell. 2005;120:747-59.

4. El-Deiry WS. The role of p53 in chemosensitivity and radiosensitivity. Oncogene. 2003;22:7486-95.

5. Steele JP, Klabatsa A. Chemotherapy options and new advances in malignant pleural mesothelioma. Ann Oncol. 2005;16:345-51.

6. Vogelzang NJ, Rusthoven JJ, Symanowski J, Denham C, Kaukel E, Ruffie P, et al. Phase III study of pemetrexed in combination with cisplatin versus cisplatin alone in patients with malignant pleural mesothelioma. J Clin Oncol. 2003;21: 2636-44.

7. van Meerbeeck JP, Gaafar R, Manegold C, Van Klaveren RJ, Van Marck EA, Vincent M, et al. Randomized phase III study of cisplatin with or without raltitrexed in patients with malignant pleural mesothelioma: an intergroup study of the European Organisation for Research and Treatment of Cancer Lung Cancer Group and the National Cancer Institute of Canada. J Clin Oncol. 2005;23: 6881-9.

8. Chahinian AP, Antman K, Goutsou M, Corson JM, Suzuki Y, Modeas C, et al. Randomized phase II trial of cisplatin with mitomycin or doxorubicin for malignant mesothelioma by the Cancer and Leukemia Group B. J Clin Oncol. 1993;11: 1559-65.

9. Bellacosa A, Kumar CC, Di Cristofano A, Testa JR. Activation of AKT kinases in cancer: implications for therapeutic targeting. Adv Cancer Res. 2005;94: 29-86.

10. Altomare DA, You H, Xiao GH, Ramos-Nino ME, Skele KL, De Rienzo A, et al. Human and mouse mesotheliomas exhibit elevated AKT/PKB activity, which can be targeted pharmacologically to inhibit tumor cell growth. Oncogene. 2005;24:6080-9.
11. Ramos-Nino ME, Testa JR, Altomare DA, Pass HI, Carbone M, Bocchetta M, et al. Cellular and molecular parameters of mesothelioma. $J$ Cell Biochem. 2006;98:723-34.

12. Brown EJ, Albers MW, Shin TB, Ichikawa K, Keith CT, Lane WS, et al. A mammalian protein targeted by G1-arresting rapamycin-receptor complex. Nature. 1994;369:756-8.

13. Kunz J, Henriquez R, Schneider U, Deuter-Reinhard M, Movva NR, Hall MN. Target of rapamycin in yeast, TOR2, is an essential phosphatidylinositol kinase homolog required for G1 progression. Cell. 1993;73:585-96.

14. Shi Y, Frankel A, Radvanyi LG, Penn LZ, Miller RG, Mills GB. Rapamycin enhances apoptosis and increases sensitivity to cisplatin in vitro. Cancer Res. 1995; 55:1982-8.

15. Vignot S, Faivre S, Aguirre D, Raymond E. mTOR-targeted therapy of cancer with rapamycin derivatives. Ann Oncol. 2005;16:525-37.

16. Wendel HG, De Stanchina E, Fridman JS, Malina A, Ray S, Kogan S, et al. Survival signalling by Akt and eIF4E in oncogenesis and cancer therapy. Nature. 2004;428:332-7.

17. Yan H, Frost P, Shi Y, Hoang B, Sharma S, Fisher M, et al. Mechanism by which mammalian target of rapamycin inhibitors sensitize multiple myeloma cells to dexamethasone-induced apoptosis. Cancer Res. 2006;66:2305-13.

18. Gordon GJ, Mani M, Maulik G, Mukhopadhyay L, Yeap BY, Kindler HL, et al. Preclinical studies of the proteasome inhibitor bortezomib in malignant pleural mesothelioma. Cancer Chemother Pharmacol. 2008;61:549-58.

19. Demetri GD, Zenzie BW, Rheinwald JG, Griffin JD. Expression of colony-stimulating factor genes by normal human mesothelial cells and human malignant mesothelioma cells lines in vitro. Blood. 1989;74:940-6.

20. Kern DH, Weisenthal LM. Highly specific prediction of antineoplastic drug resistance with an in vitro assay using suprapharmacologic drug exposures. $J$ Natl Cancer Inst. 1990;82:582-8.

21. Tanigawa N, Morimoto H, Dohmae N, Shimomatsuya T, Takahashi K, Muraoka R. In vitro growth ability and chemosensitivity of gastric and colorectal cancer cells assessed with the human tumour clonogenic assay and the thymidine incorporation assay. Eur J Cancer. 1992;28:31-4.

22. Sawyers CL. Will mTOR inhibitors make it as cancer drugs? Cancer Cell. 2003;4: 343-8.

23. O'Reilly KE, Rojo F, She QB, Solit D, Mills GB, Smith D, et al. mTOR inhibition induces upstream receptor tyrosine kinase signaling and activates Akt. Cancer Res. 2006;66:1500-8.

24. Gingras AC, Raught B, Gygi SP, Niedzwiecka A, Miron M, Burley SK, et al. Hierarchical phosphorylation of the translation inhibitor 4E-BP1. Genes Dev. 2001; 15:2852-64.

25. Barbone D, Yang TM, Morgan JR, Gaudino G, Broaddus VC. mTOR contributes to the acquired apoptotic resistance of human mesothelioma multicellular spheroids. J Biol Chem. 2008;283:13021-30. 